\author{
Jacques COLIN \\ Besançon Observatory \\ 25000 BESANCON (France)
}

\begin{abstract}
We determine if the orbits of the galactic globular clusters can be circular, using a spherical logarithmic potential. If they are not, we calculate their perigalactic distances and we deduce a pseudo-eccentricity. Using a numerical and geometrical method, we obtain the minimum of all the possible pseudo-eccentricities for each cluster. The corresponding perigalactic distances are compared with the values given by Innanen et al. (1983) with a different method.
\end{abstract}

In this paper we consider that the galactic potential can be represented by a logarithmic function. This is consistent with the observed flat rotation curve, and the existence of a massive halo. In such a model, the visible disk will not, to first order, modify the orbital characteristics of the globular clusters.

Using this potential and the radial velocity $V_{R}$ given by Harris and Racine (1979), we consider all the orbits compatible with the model and we select those which have the minimum pseudo-eccentricity.

Thus, we obtain a sample of globular clusters whose orbits are not circular and we compare the corresponding perigalactic distances with those given by Innanen et al. (1983).

If $V_{\text {Rot }}$ is the constant rotational velocity corresponding to the flat rotation curve, $V_{T}$, the unknown tangential velocity and $V_{S}$ the unknown spatial velocity, the orbit is circular if:

$$
\mathrm{V}_{\mathrm{S}}=\mathrm{V}_{\text {Rot }}
$$

This is possible only if $\left.V_{\text {Rot }}\right)\left(v_{R} / \sin B\right)$ where $B$ is the angle under which is seen the distance Sun-Galactic center.

If $V_{\text {Rot }}<\left(v_{R} / \sin B\right)$, the orbit cannot be circular, thus there exists a perigalactic and an apogalactic distance $r_{p}$ and $r_{a}$, and we define a pseudo-eccentricity:

$$
e=\left(r_{a}-r_{p}\right) /\left(r_{a}+r_{p}\right)
$$

J. Goodman and P. Hut (eds.), Dynamics of Star Clusters, 309-312.

(C) 1985 by the IAU. 


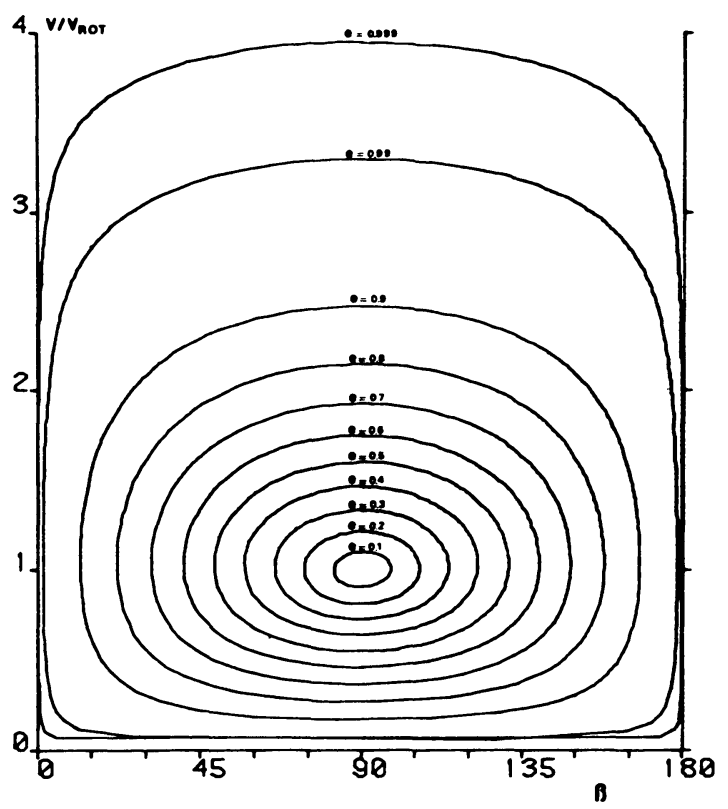

Figure 1 - Eccentricity of an orbit in a Iogarithmic potential as a function of the velocity vector define by the modulus $V / V_{R o t}$ and the direction $B$. This diagram is independant of the distance to the galactic center.

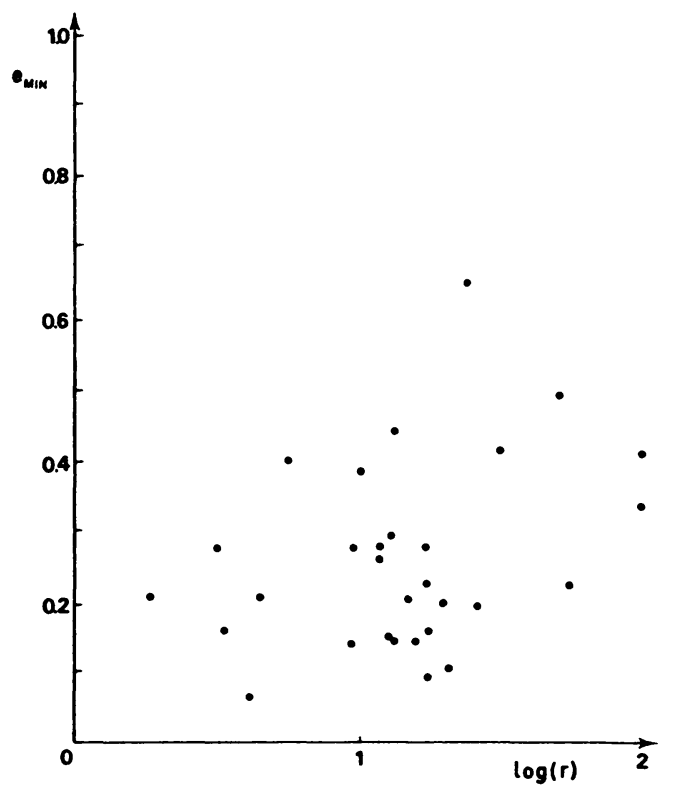

Figure 2 - Minimum of the eccentricity for 30 globular clusters as a function of the distance $r$. For the other clusters, the minimum is zero. 
By varying $\mathrm{V}_{\mathrm{T}}$ we explore all the possible orbits and the corresponding pseudo- eccentricities. For that, we use two methods, a geometrical and a numerical one.

\section{NUMERICAL METHOD}

For each cluster we compute a sample of possible orbit by varying $\mathrm{V}_{\mathrm{T}}$. We represent the variation of e relative to $V_{T}$ and we obtain a minimum for the eccentricity.

\section{GEOMETRICAL METHOD}

In a logarithmic potential it is possible to determine the eccentricity of the orbit of a test particle, when its velocity vector is known (Colin and Puel, unpublished).

On Figure 1 we show the relation between the components of the velocity vector (modulus $V$ and direction $B$ ) and the ellipticity of the orbits. This diagram must be compared to the Bottlinger diagram but here the distance to the galactic center is ignored. With this diagram the radial velocity and the position of the cluster it is possible to determine the minimum of the pseudo-eccentrictry of a globular cluster. But we don't obtain $r_{a}$ and $r_{p}$ with this method.

We obtain 30 globular clusters for which the pseudo-eccentricity is different from zero. We also obtain the ratio $\left(R_{p} / R\right)$ of the pericenter distance to the present distance of the clusters. These values may be compared with those of Innanen et al. (1982) but the significance is not exactly the same. For Innanen et al. the values are deduced from the observations and must correspond to the true orbits. In our case the given values correspond to the maximum possible value, but not necessarily to the real one. Thus, the values of Innanen et al. (1982) must not be greater than ours.

\section{DISCUSSION}

From a geometrical and a mechanical point of vue, $60 \%$ of the globular clusters may have a circular orbit, if the galaxy is represented by a spherical logarithmic potential. If we exclude the $R_{p} / R>1$, most of the values of $R_{p} / R$ given by Innanen et al. (1982) agree with ours, they don't exceed our values that normally are maximum values.

It also seems that the minimum eccentricity grows with distance. Figure 2 shows this peculiarity already pointed out by Rastorguev and Surdin (1980) for a different parameter $\left(e_{\min }=\left(R-R_{p}\right) /\left(R+R_{p}\right)\right.$. 


\section{REFERENCES}

Harris, W.E. and Racine, R. (1979) Ann. Rev. Astron. Astrophys.17, 241. Innanen, K.A., Harris, W.E., Webbink, R.F. (1983) Astron. J. 88, 338. Rastorguev, A.S. and Surdin, V.G. (1980) Astron. Tsirk., $\mathrm{N}^{\circ} 1102$. 